Piezo1 alters inflammatory signalling which may have implications on PC pathophysiology. We plan to investigate this observation further to understand how Piezo1-mediated signalling influences PC-EC communication in exercise and general health.

Conflict of Interest no

\section{BS3 NOVEL STRATEGY USING HESC-DERIVED CARDIOMYOCYTES TO EXPLORE THE CRITICAL IMPORTANCE OF THE APELIN RECEPTOR IN THE CARDIOVASCULAR SYSTEM}

\begin{abstract}
${ }^{1}$ Robyn Macrae, ${ }^{1}$ Maria Colzani, ' $E m m a$ Robinson, 'Semih Bayraktar, ${ }^{1}$ Thomas Williams, ${ }^{1}$ Rhoda Kuc, 'Janet Maguire, 'Sanjay Sinha, ${ }^{1}$ Anthony Davenport. ' University of Cambridge, Cambridge, UK; ${ }^{2}$ University of Colorado
\end{abstract}

10.1136/heartjnl-2021-BCS.201

Introduction Apelin receptor activation promotes beneficial vasodilation and positive inotropy ${ }^{1}$ and is critical for early cardiac development. ${ }^{2}$ In cardiovascular disease states, including heart failure, expression of apelin receptor endogenous peptide ligands, apelin and Elabela, are downregulated. ${ }^{1}$ We aim to use human embryonic stem cell (hESC)-derived cardiomyocytes (hESC-CMs) as a model to interrogate the role of the apelin receptor in the cardiovascular system and the potential of targeting the receptor therapeutically. Here, we have generated a system to inducibly knockdown the apelin receptor in hESCCMs to examine the effects on differentiation of hESC to cardiomyocyte, and the effects on function of the resulting hESC-CMs.

Methods A short hairpin RNA based apelin receptor (shAPLNR) inducible knockdown system was generated in pluripotent hESCs, utilising the sOPTiKD system as described previously, ${ }^{3}$ before differentiating to hESC-CMs. Inclusion of tetracycline in the culture medium was used to induce knockdown, with knockdown efficiency determined by qRT-PCR and radioligand binding. RNA-sequencing analysis and a panel of phenotypic assays were performed to examine effects of apelin receptor knockdown on cardiomyocyte differentiation and function.

Results We have previously demonstrated that hESC-derived cardiomyocytes express the apelinergic system at a similar level to adult cardiomyocytes (Bmax hESC-CMs $21 \mathrm{fmol} / \mathrm{mg}$ versus adult $14 \mathrm{fmol} / \mathrm{mg}$ ). Apelin receptor expression was significantly reduced in hESCs and hESC-CMs by tetracycline inclusion at both RNA and protein level $(84.2 \% \pm 4.3$ and $82.8 \% \pm 3.9$ compared to control in hESCs and hESC-CMs, respectively). Interestingly, efficiency of differentiation to cardiomyocyte was reduced compared to control cells $(77.8 \% \pm$ 5.5 cardiomyocyte control vs $22.2 \% \pm 7.4$ cardiomyocyte knockdown). RNA-sequencing analysis revealed 272 differentially expressed genes involved in pathways related to electrophysiological signalling, adhesion and the cytoskeleton.

Discussion We have successfully shown knockdown of apelin receptor expression in hESC-CMs. To our knowledge, this is the first use of this system to knockdown expression of a GPCR. This system allows detailed characterisation of apelin receptor activation in cardiovascular development and disease pathogenesis, providing an innovative approach to develop strategies to target the apelin receptor therapeutically in cardiovascular disease, where novel treatments are urgently needed. Conflict of Interest None
REFERENCES

1. Yang et al. Circulation 2017;135:1160-1173.

2. Chng et al. Developmental Cell 2013;27(6):672-680.

3. Bertero et al. Development 2016;143:4405-4418.

\section{BS4 MODELLING OF STROKE RISK: AN EPIGENETIC AND IN VITRO STUDY}

${ }^{1}$ Natalia Cullell, ${ }^{2}$ Cristina Gallego-Fábrega, ${ }^{2}$ Jara Cárcel, ${ }^{2}$ Elena Muiño, ${ }^{2}$ Laia Llucià-Carol, ${ }^{2}$ Miquel Lledós, ${ }^{3}$ Robert Beal, ${ }^{3}$ Stephen White, ${ }^{4}$ Manel Esteller, ${ }^{4}$ Manuel Castro de Moura, ${ }^{5}$ Jerzy Krupinski, ${ }^{6}$ Israel Fernández-Cadenas. ${ }^{1}$ Fundació Docència i Recerca MutuaTerrassa / Biomedical Research Institute Sant Pau, Terrassa, Spain; ${ }^{2}$ Biomedical Research Institute Sant Pau; ${ }^{3}$ Manchester Metropolitan University; ${ }^{4}$ Josep Carreras Leukaemia Research Institute; ${ }^{5}$ Fundació Docència i Recerca MutuaTerrassa; ${ }^{6}$ Biomedical Research Institute Sant Pau, Fundació Docència i Recerca MutuaTerrassa

\subsection{6/heartjnl-2021-BCS.202}

Background and Aims 35 loci have been associated with stroke in Genome-Wide association studies (GWAS). We previously found an altered DNA methylation pattern in one gene identified in GWAS to be associated with stroke risk (ZFHX3). DNA methylation in this gene was causally associated with atherothrombotic stroke. Our aim is to determine whether genes associated with stroke risk in GWAS are also linked with stroke susceptibility through epigenetic regulation. Moreover, to study the implication of these genes in the atherosclerotic process using an in vitro model.

Methods DNA methylation was assessed in 253 ischemic stroke patients and 43 controls using the Infinium $450 \mathrm{KBead}-$ Chip and EPICBeadChip. We selected all the CpG-sites located in the 35 loci previously associated with stroke in the Megastroke + Uk Biobank cohorts. The significant associations were evaluated in an in vitro model of human coronary artery endothelial cells exposed to normal laminar flow (LSS) and atherogenic flow environments: oscillatory (OSS) and elevated laminar shear stress (ESS).

Results $134 \mathrm{CpG}$-sites located in 27 different loci were associated with stroke $(\mathrm{p}<0.05)$ and $8 \quad \mathrm{CpG}$-sites remained significant after Bonferroni adjustment. These CpG-sites corresponded to 6 different genes: ZFHX3, SH2B3, SMARCA4, TSPAN2, ILF3 and CDK6. All of them presented hypomethylation in stroke patients compared with controls. We found a significant increased expression of ZFHX3 and CDK6 in ESS compared to LSS. Expression of ILF3 was $60 \%$ higher in OSS and 40\% lower in ESS compared to LSS. The phosphorylation status of ZFHX3 and SMARCA4 was found to be increased in the OSS environment.

Conclusions Our findings indicate that epigenetic regulation of genes that are risk factors for stroke is associated with stroke susceptibility.

Conflict of Interest No

\section{BS5 EFFECTS OF HYPOKALAEMIA ON ELECTROPHYSIOLOGICAL AND CALCIUM HANDLING OF HUMAN PLURIPOTENT STEM CELL-DERIVED CARDIAC ANISOTROPIC SHEETS}

Bimal Gurung. The Chinese University of Hong Kong, Sha Tin, Hong Kong (China)

10.1136/heartjnl-2021-BCS.203

Introduction Hypokalaemia, defined as extracellular concentration $[\mathrm{K}+]$ below $3.5 \mathrm{mM}$, can cause cardiac arrhythmias by 

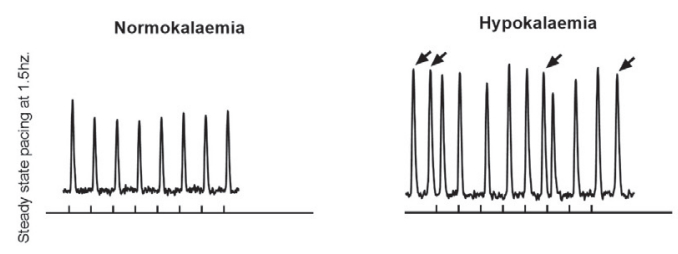

(A)
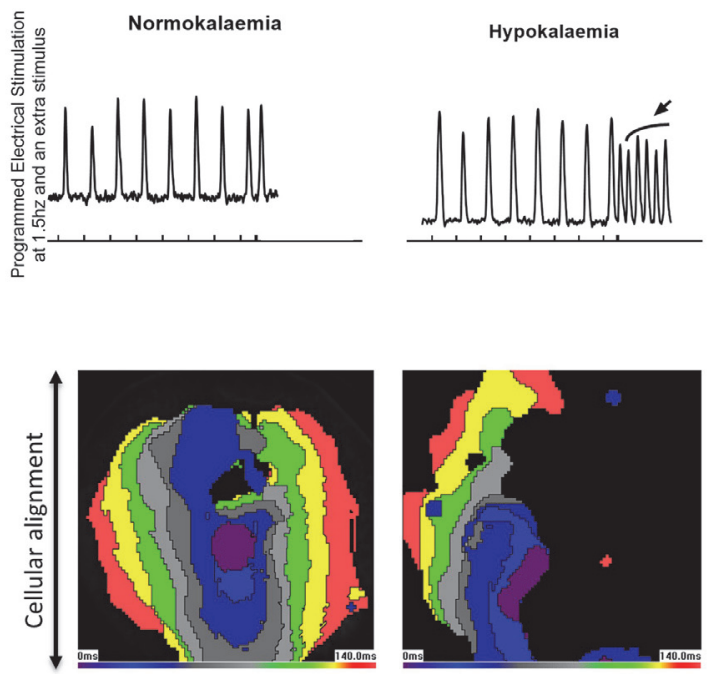

(B)

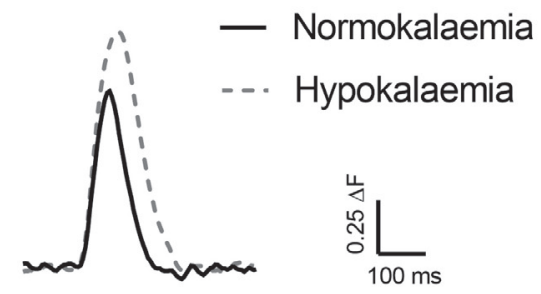

岇

(D)

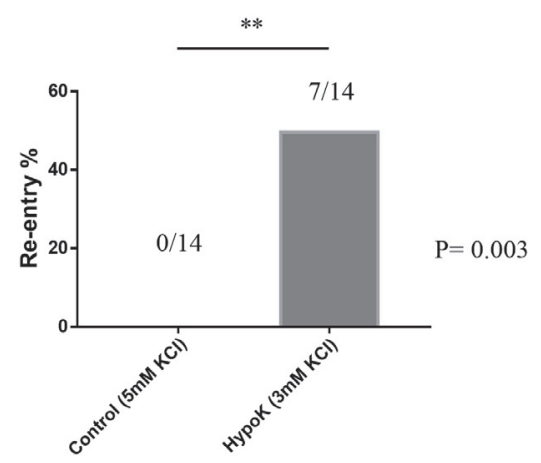

\section{Abstract BS5 Figure 1}

triggered or reentrant mechanisms. Whilst these effects have been reported in animal and human stem cell-based models, to date there has been no investigation in more complex structures such as the human ventricular cardiac anisotropic sheet (hvCAS). Here, we investigated arrhythmogenicity, electrophysiological and calcium transient changes induced by hypokalaemia using this bioengineered platform.

Methods An optical mapping technique was applied on hvCAS derived from human pluripotent stem cells to visualize electrophysiological and calcium transient changes under normokalaemic $(5 \mathrm{mM} \mathrm{KCl})$ and hypokalaemic $(3 \mathrm{mM} \mathrm{KCl})$ conditions.

Results Hypokalaemia significantly increased the proportion of preparations showing spontaneous arrhythmias from $0 / 14$ to $7 / 14$ (Fisher's exact test, $\mathrm{P}=0.003$ ) (figure 1D). Hypokalaemia reduced longitudinal conduction velocity (CV) from 7.81 to $7.18 \mathrm{cms}-1$ ( $\mathrm{n}=9-7 ; \mathrm{P}=0.036)$, transverse $\mathrm{CV}$ from 5.72 to $4.69 \mathrm{cms}-1$ ( $\mathrm{n}=12-11 ; \mathrm{P}=0.030)$, prolonged action potential at $90 \%$ repolarization (APD90) from 83.46 to $97.45 \mathrm{~ms}(\mathrm{n}=$ 13-15; $\mathrm{P}<0.001)$, increased action potential amplitude from 0.888 to $1.195 \Delta \mathrm{F}(\mathrm{n}=12-14 ; \mathrm{P}<0.001)$ and calcium transient amplitude from 0.76 to $1.37 \Delta \mathrm{F}$ ( $\mathrm{n}=12-13 ; \mathrm{P}<0.001)$, and shortened effective refractory periods from 233 to 173 ms ( $\mathrm{n}=16-14 ; \mathrm{P}=0.038)$. Figure $1(\mathrm{~A}),(\mathrm{B})$ and $(\mathrm{C})$ show the representative tracings and isochronal maps of normokalaemic and hypokalaemic samples.

Conclusions Hypokalaemia exerts pro-arrhythmic effects on hvCAS, which are associated with alterations in conduction velocity, repolarization, refractoriness and calcium handling. These preparations can provide a useful platform for investigating electrophysiological substrates and for arrhythmia screening.
Conflict of Interest None

\section{BS6 COHERENT VORTEX FLOW IN THE ADULT RIGHT ATRIUM}

${ }^{1}$ Lian Gan, ${ }^{1}$ Peter Dewhurst, ${ }^{2}$ Louise Coats, ${ }^{3}$ Jehill Parikh, ${ }^{3}$ Kieren Hollingsworth. ${ }^{1}$ Durham University, Durham, UK; ${ }^{2}$ Newcastle University and Freeman Hospital; ${ }^{3}$ Newcastle University

\subsection{6/heartjnl-2021-BCS.204}

Brief introduction: In healthy adults, the right atrium (RA) serves as a reservoir for the systemic flow return from the superior vena cava (SVC) and inferior vena cava (IVC), preparing the two flows to be transferred to the right ventricle (RV) and pulmonary circulation. This study aims to quantify the haemodynamics of the RA and the associated SVC and IVC inflows, which have not been fully understood to date.

Basic Methods Eighteen adults with structurally normal hearts underwent 4D flow magnetic resonance imaging. The cardiac cycle was resolved to 20 temporal phases with a spatial resolution of $3 \times 3 \times 3 \mathrm{~mm}$ cube. Analysis included objective visualisation of the flow structures in the RA identified by three different vortex identification criteria, kinetic energy (KE), enstrophy and dissipation. KE and helicity flux were also assessed in both caval veins.

Main Results Vortex identification methods confirmed that in the majority of participants the blood flow from the caval veins filling the RA during ventricular systole is not chaotic, but rather forms an organised pattern of a single coherent forward turning vortex structure. Thirteen participants displayed 